\title{
On the distribution of the van der Corput sequence in arbitrary base
}

\author{
Bence Borda \\ Department of Mathematics, Rutgers University \\ 110 Frelinghuysen Road, Piscataway, NJ-08854, USA \\ Email: bordabence85@gmail.com
}

Keywords: van der Corput sequence, $L^{p}$ discrepancy, central limit theorem, large deviations Mathematics Subject Classification (2010): 11K31, 11K38, 60F05, 60F10

\begin{abstract}
A central limit theorem with explicit error bound, and a large deviation result are proved for a sequence of weakly dependent random variables of a special form. As a corollary, under certain conditions on the function $f:[0,1] \rightarrow \mathbb{R}$ a central limit theorem and a large deviation result are obtained for the sum $\sum_{n=0}^{N-1} f\left(x_{n}\right)$, where $x_{n}$ is the base $b$ van der Corput sequence for an arbitrary integer $b \geq 2$. Similar results are also proved for the $L^{p}$ discrepancy of the same sequence for $1 \leq p<\infty$. The main methods used in the proofs are the Berry-Esseen theorem and Fourier analysis.
\end{abstract}

\section{Introduction}

For an integer $b \geq 2$ the base $b$ van der Corput sequence $x_{n}$ is defined the following way. If the base $b$ representation of the integer $n \geq 0$ is $n=\sum_{i=1}^{m} a_{i} b^{i-1}$ for some digits $a_{i} \in\{0,1, \ldots, b-1\}$, then

$$
x_{n}=\sum_{i=1}^{m} \frac{a_{i}}{b^{i}} .
$$

The main importance of this sequence is that it is of low discrepancy. Indeed, the discrepancy function of the base $b$ van der Corput sequence

$$
\Delta_{N}(x)=\left|\left\{0 \leq n<N: x_{n}<x\right\}\right|-N x,
$$

defined for nonnegative integers $N$, and $x \in[0,1]$, satisfies

$$
0 \leq \Delta_{N}(x) \leq \frac{b}{4} \log _{b} N+b
$$


The precise value of

$$
\limsup _{N \rightarrow \infty} \frac{\sup _{x \in[0,1]} \Delta_{N}(x)}{\log N}
$$

in terms of the base $b$ was found by Faure (4] Theorem 1, Theorem 2 and Sections 5.5.1-5.5.3).

In this article we study the random aspects of the base $b$ van der Corput sequence. Let

$$
\Phi(\lambda)=\int_{-\infty}^{\lambda} \frac{1}{\sqrt{2 \pi}} e^{-\frac{x^{2}}{2}} \mathrm{~d} x
$$

denote the distribution function of the standard normal distribution. Our main result is that the sum

$$
S(N)=\sum_{n=0}^{N-1}\left(\frac{1}{2}-x_{n}\right)
$$

satisfies the following central limit theorem.

Theorem 1. Let $x_{n}$ be the base $b$ van der Corput sequence, where $b \geq 2$ is an arbitrary integer. Then for any integer $M>b^{2}$ and any real number $\lambda$ we have

$$
\frac{1}{M}\left|\left\{0 \leq N<M: \frac{S(N)-c(b) \log _{b} N}{\sqrt{d(b) \log _{b} N}}<\lambda\right\}\right|=\Phi(\lambda)+O\left(\frac{\sqrt[4]{\log \log _{b} M}}{\sqrt[4]{\log _{b} M}}\right)
$$

where $c(b)=\frac{b^{2}-1}{12 b}$ and $d(b)=\frac{b^{4}+120 b^{3}-480 b^{2}+600 b-241}{720 b^{2}}$. The implied constant in the error term is absolute.

The following large deviation result complements Theorem 1.

Theorem 2. Let $x_{n}$ be the base $b$ van der Corput sequence, where $b \geq 2$ is an arbitrary integer. For any integer $M>b$ and any real number $\lambda \geq 3$ we have

$$
\begin{aligned}
\frac{1}{M}\left|\left\{0 \leq N<M:\left|S(N)-\frac{b^{2}-1}{12 b} \log _{b} M\right| \geq 25 \lambda b \sqrt{\log _{b} M+1}\right\}\right| & \\
& \leq \frac{4 \sqrt{\lambda}}{e^{\sqrt{\lambda}-1}-2}+\frac{1}{b^{\sqrt{\log _{b} M}-2}}
\end{aligned}
$$


Since

$$
\int_{0}^{1} \Delta_{N}(x) \mathrm{d} x=S(N)
$$

we have that $S(N)=O\left(b \log _{b} M\right)$, therefore Theorem 2 is meaningful only when applied with $\lambda=O\left(\sqrt{\log _{b} M}\right)$. Note that for all such values of $\lambda$ the error term $\frac{1}{b \sqrt{\log _{b} M-2}}$ is of smaller order of magnitude than $\frac{4 \sqrt{\lambda}}{e^{\sqrt{\lambda}-1}-2}$. The question of whether the upper bound in Theorem 2 can be improved to $O\left(e^{-d \lambda}\right)$ or to $O\left(e^{-d \lambda^{2}}\right)$ for some constant $d>0$ is left open.

Observation (1) gives the idea that the sum $S(N)$ is related to the $L^{p}$ norm

$$
\left\|\Delta_{N}\right\|_{p}=\left(\int_{0}^{1}\left|\Delta_{N}(x)\right|^{p} \mathrm{~d} x\right)^{\frac{1}{p}}
$$

of the discrepancy function. As simple corollaries to Theorem 1 and Theorem 2 we thus obtain that $\left\|\Delta_{N}\right\|_{p}$ satisfies the same central limit theorem and large deviation result as $S(N)$.

Theorem 3. Let $x_{n}$ be the base $b$ van der Corput sequence, where $b \geq 2$ is an arbitrary integer. Let $1 \leq p<\infty$ be an arbitrary real. Then for any integer $M>b^{2}$ and any real number $\lambda$ we have

$$
\frac{1}{M}\left|\left\{0 \leq N<M: \frac{\left\|\Delta_{N}\right\|_{p}-c(b) \log _{b} N}{\sqrt{d(b) \log _{b} N}}<\lambda\right\}\right|=\Phi(\lambda)+O\left(\frac{\sqrt[4]{\log \log _{b} M}}{\sqrt[4]{\log _{b} M}}\right)
$$

where $c(b)=\frac{b^{2}-1}{12 b}$ and $d(b)=\frac{b^{4}+120 b^{3}-480 b^{2}+600 b-241}{720 b^{2}}$. The implied constant in the error term depends only on $p$.

Theorem 4. Let $x_{n}$ be the base $b$ van der Corput sequence, where $b \geq 2$ is an arbitrary integer. Let $1 \leq p<\infty$ be an arbitrary real. There exists a positive constant $A$ depending only on $p$ such that for any integer $M>b$ and any real number $\lambda \geq 1$ we have

$$
\frac{1}{M}\left|\left\{0 \leq N<M:\left|\left\|\Delta_{N}\right\|_{p}-\frac{b^{2}-1}{12 b} \log _{b} N\right| \geq A \lambda b \sqrt{\log _{b} N}\right\}\right| \leq e^{-\sqrt{\lambda}} .
$$

Similar central limit theorems concerning the distribution of the van der Corput sequence have already appeared in the literature. In [3] Theorem 3 is proved in the special case when $b=2$ with an error term $o(1)$ of unspecified order of magnitude. 
In Section 1.3 of [1 Theorem 1 is proved, again in the special case $b=2$, with an error term $O\left(\frac{\log \log M}{\sqrt[10]{\log M}}\right)$. Our proof of Theorem 1 is the generalization of the proof in Section 1.3 of [1]. In a doctoral dissertation ([7] Theorem 4.1.1.) a central limit theorem for the supremum norm $\left\|\Delta_{N}\right\|_{\infty}$ of the discrepancy function in the case of an arbitrary base $b \geq 2$, similar to Theorem 3 is proved. The main difference is that $c(b)$ is to be replaced by $c_{\infty}(b)=\frac{2 b-1}{12}$ and $d(b)$ is to be replaced by

$$
d_{\infty}(b)=\frac{4 b^{7}-10 b^{6}+10 b^{5}+14 b^{4}-77 b^{3}+127 b^{2}-68 b+8}{720 b^{2}(b-1)^{2}(b+1)} .
$$

Moreover, the theorem is stated only in the special case when $M$ is a power of the base $b$, and the error term is of an unspecified order of magnitude $o(1)$. In [3] and [7] central limit theorems for various generalizations of the van der Corput sequence are also studied. Large deviation results have not yet been obtained.

Finally, we give a method to generalize Theorem 1 and Theorem 2 for sums of the form $\sum_{n=0}^{N-1} f\left(x_{n}\right)$, where the function $f:[0,1] \rightarrow \mathbb{R}$ is sufficiently nice, and $x_{n}$ is the base $b$ van der Corput sequence. Since the discrepancy satisfies

$$
\sup _{x \in[0,1]}\left|\Delta_{N}(x)\right|=O\left(b \log _{b} N\right),
$$

the Koksma inequality ([5] Chapter 2 Theorem 5.1) implies that if $f:[0,1] \rightarrow \mathbb{R}$ is of bounded variation, then

$$
\sum_{n=0}^{N-1} f\left(x_{n}\right)=N \int_{0}^{1} f(x) \mathrm{d} x+O(\log N),
$$

as $N \rightarrow \infty$, with an implied constant depending only on $b$ and the total variation of $f$. Under more restrictive assumptions on the function $f$ the error term actually satisfies a central limit theorem and a large deviation result. The following proposition reduces the problem of studying the distribution of $\sum_{n=0}^{N-1} f\left(x_{n}\right)$ to that of $S(N)$.

Proposition 5. Let $f:[0,1] \rightarrow \mathbb{R}$ be twice differentiable with $f^{\prime \prime} \in L^{1}([0,1])$, and let $x_{n}$ denote the base $b$ van der Corput sequence, where $b \geq 2$ is an arbitrary integer. For any integer $N>0$ we have

$$
\left|\sum_{n=0}^{N-1} f\left(x_{n}\right)-N \int_{0}^{1} f(x) \mathrm{d} x+(f(1)-f(0)) S(N)\right| \leq \frac{b}{3}\left\|f^{\prime \prime}\right\|_{1} .
$$

The natural interpretation of the quantity $f(1)-f(0)$ is that the periodic extension of $f$ on $\mathbb{R}$ with period 1 has jumps of this size. 
In Section 2 we derive the normalizing factors $c(b)$ and $d(b)$ of Theorem 1 . Section 3 is devoted to the proofs of Theorem 1 and Theorem 2 , while the proofs of Theorem 3, Theorem 4 and Proposition 5 are given in Section 4 .

\section{The expected value and the variance of $S(N)$}

We start by deriving a formula for the sum $S(N)$ in terms of the base $b$ digits of $N$ as follows.

Proposition 6. Let $b \geq 2$ be an integer and let $N=\sum_{i=1}^{m} a_{i} b^{i-1}$ be the base $b$ representation of an integer $N \geq 0$, where $a_{i} \in\{0,1, \ldots, b-1\}$. Then

$$
S(N)=\sum_{i=1}^{m} \frac{(b+1) a_{i}-a_{i}^{2}}{2 b}-\sum_{1 \leq i<j \leq m} \frac{a_{i} a_{j}}{b^{j-i+1}} .
$$

Proof. By splitting the sum $S(N)$ we get

$$
S(N)=\sum_{n=0}^{a_{m} b^{m-1}-1}\left(\frac{1}{2}-x_{n}\right)+\sum_{n=a_{m} b^{m-1}}^{N-1}\left(\frac{1}{2}-x_{n}\right) .
$$

Since

$$
\left\{x_{n}: 0 \leq n<a_{m} b^{m-1}\right\}=\left\{\frac{k}{b^{m-1}}+\frac{a}{b^{m}}: 0 \leq k<b^{m-1}, \quad 0 \leq a<a_{m}\right\},
$$

we obtain that the first sum in (2) is

$$
\sum_{n=0}^{a_{m} b^{m-1}-1}\left(\frac{1}{2}-x_{n}\right)=\sum_{k=0}^{b^{m-1}-1} \sum_{a=0}^{a_{m}-1}\left(\frac{1}{2}-\frac{k}{b^{m-1}}-\frac{a}{b^{m}}\right)=\frac{(b+1) a_{m}-a_{m}^{2}}{2 b} .
$$

To compute the second sum in (2) note that for any $a_{m} b^{m-1} \leq n<N$ the first base $b$ digit of $n$ is $a_{m}$, and hence

$$
x_{n}=x_{n-a_{m} b^{m-1}}+\frac{a_{m}}{b^{m}} .
$$

Therefore by reindexing the sum we obtain

$$
\begin{aligned}
\sum_{n=a_{m} b^{m-1}}^{N-1}\left(\frac{1}{2}-x_{n}\right) & =\sum_{n=0}^{N-a_{m} b^{m-1}-1}\left(\frac{1}{2}-x_{n}-\frac{a_{m}}{b^{m}}\right) \\
& =S\left(N-a_{m} b^{m-1}\right)-\frac{a_{m}}{b^{m}}\left(N-a_{m} b^{m-1}\right) .
\end{aligned}
$$


Using the base $b$ representation of $N$ we thus find the recursion

$$
S\left(\sum_{i=1}^{m} a_{i} b^{i-1}\right)=\frac{(b+1) a_{m}-a_{m}^{2}}{2 b}-\sum_{i=1}^{m-1} \frac{a_{i} a_{m}}{b^{m-i+1}}+S\left(\sum_{i=1}^{m-1} a_{i} b^{i-1}\right) .
$$

Applying the recursion (3) $m$ times finishes the proof.

If $N$ is a random variable uniformly distributed in $\left\{0,1, \ldots, b^{m}-1\right\}$ for some integers $b \geq 2$ and $m \geq 1$, then the base $b$ digits $a_{1}, \ldots, a_{m}$ of $N$ are independent random variables, each uniformly distributed in $\{0,1, \ldots, b-1\}$. Therefore Proposition 6 can be used to find the expected value and the variance of the sum $S(N)$. Here and from now on the expected value and the variance of a real valued random variable $X$ are denoted by $\mathrm{E}(X)$ and $\operatorname{Var}(X)$, respectively.

Proposition 7. Let $N$ be a random variable which is uniformly distributed in $\left\{0,1, \ldots, b^{m}-1\right\}$ for some integers $b \geq 2$ and $m \geq 1$. Then

$$
\begin{gathered}
\left|\mathrm{E}(S(N))-\frac{b^{2}-1}{12 b} m\right| \leq \frac{1}{4}, \\
\operatorname{Var}(S(N))=\frac{b^{4}+120 b^{3}-480 b^{2}+600 b-241}{720 b^{2}} m+O(b) .
\end{gathered}
$$

The implied constant in the error term is absolute.

Proof. Using the independence of the base $b$ digits $a_{1}, \ldots, a_{m}$ of $N$, from Proposition 6 we get that the expected value of $S(N)$ is

$\mathrm{E}(S(N))=\sum_{i=1}^{m} \frac{(b+1) \mathrm{E}\left(a_{i}\right)-\mathrm{E}\left(a_{i}^{2}\right)}{2 b}-\sum_{1 \leq i<j \leq m} \frac{\mathrm{E}\left(a_{i}\right) \mathrm{E}\left(a_{j}\right)}{b^{j-i+1}}=\frac{b^{2}-1}{12 b} m+\frac{1}{4}-\frac{1}{4 b^{m}}$.

To find the variance of $S(N)$, first let us use the independence of $a_{1}, \ldots, a_{m}$ again to obtain

$$
\operatorname{Var}\left(\sum_{i=1}^{m} \frac{(b+1) a_{i}-a_{i}^{2}}{2 b}\right)=\sum_{i=1}^{m} \operatorname{Var}\left(\frac{(b+1) a_{i}-a_{i}^{2}}{2 b}\right)=\frac{b^{4}+55 b^{2}-56}{720 b^{2}} m .
$$

Now consider 
$\operatorname{Var}\left(\sum_{1 \leq i<j \leq m} \frac{a_{i} a_{j}}{b^{j-i+1}}\right)=\sum_{\substack{1 \leq i_{1}<j_{1} \leq m \\ 1 \leq i_{2}<j_{2} \leq m}}\left(\mathrm{E}\left(a_{i_{1}} a_{j_{1}} a_{i_{2}} a_{j_{2}}\right)-\frac{(b-1)^{4}}{16}\right) \frac{1}{b^{j_{1}-i_{1}+1} b^{j_{2}-i_{2}+1}}$

We will group the terms according to the size of $\left\{i_{1}, j_{1}\right\} \cap\left\{i_{2}, j_{2}\right\}$. If $\left\{i_{1}, j_{1}\right\} \cap$ $\left\{i_{2}, j_{2}\right\}$ is the empty set, then $a_{i_{1}}, a_{j_{1}}, a_{i_{2}}, a_{j_{2}}$ are independent, and therefore the contribution is zero.

If $\left\{i_{1}, j_{1}\right\} \cap\left\{i_{2}, j_{2}\right\}$ has size 1 , then

$$
\mathrm{E}\left(a_{i_{1}} a_{j_{1}} a_{i_{2}} a_{j_{2}}\right)-\frac{(b-1)^{4}}{16}=\frac{(b-1)^{3}(b+1)}{48} .
$$

Let $s>0, t>0$ and $1 \leq A \leq m-s-t$ be integers. The sum of $\frac{1}{b^{j_{1}-i_{1}+1} b^{j_{2}-i_{2}+1}}$ over all $1 \leq i_{1}<j_{1} \leq m$ and $1 \leq i_{2}<j_{2} \leq m$ such that $\left\{i_{1}, j_{1}\right\} \cup\left\{i_{2}, j_{2}\right\}=$ $\{A, A+s, A+s+t\}$ is $\frac{2}{b^{2 s+t+2}}+\frac{2}{b^{s+t+2}}+\frac{2}{b^{s+2 t+2}}$, hence we have that the contribution of this case in (5) is

$$
\begin{aligned}
\frac{(b-1)^{3}(b+1)}{48} \sum_{\substack{s, t>0 \\
s+t \leq m}}(m-s-t)\left(\frac{2}{b^{2 s+t+2}}+\frac{2}{b^{s+t+2}}+\frac{2}{b^{s+2 t+2}}\right) \\
=\frac{b^{2}+2 b-3}{24 b^{2}} m+O(1) .
\end{aligned}
$$

If $\left\{i_{1}, j_{1}\right\} \cap\left\{i_{2}, j_{2}\right\}$ has size 2 , then $i_{1}=i_{2}$ and $j_{1}=j_{2}$, and hence

$$
\mathrm{E}\left(a_{i_{1}} a_{j_{1}} a_{i_{2}} a_{j_{2}}\right)-\frac{(b-1)^{4}}{16}=\frac{\left(7 b^{2}-12 b+5\right)\left(b^{2}-1\right)}{144} .
$$

Therefore the contribution of this case in (5) is

$$
\frac{\left(7 b^{2}-12 b+5\right)\left(b^{2}-1\right)}{144} \sum_{1 \leq i<j \leq m} \frac{1}{b^{2 j-2 i+2}}=\frac{7 b^{2}-12 b+5}{144 b^{2}} m+O(1) .
$$

Altogether we find that

$$
\operatorname{Var}\left(\sum_{1 \leq i<j \leq m} \frac{a_{i} a_{j}}{b^{j-i+1}}\right)=\frac{13 b^{2}-13}{144 b^{2}} m+O(1) .
$$

Finally, it is easy to see that two times the covariance of the sums in question is 


$$
\begin{array}{r}
2 \sum_{\substack{1 \leq i_{1} \leq m \\
1 \leq i_{2}<j_{2} \leq m}} \mathrm{E}\left(\left(\frac{(b+1) a_{i_{1}}-a_{i_{1}}^{2}}{2 b}-\frac{b^{2}+3 b-4}{12 b}\right)\left(\frac{(b-1)^{2}}{4}-a_{i_{2}} a_{j_{2}}\right) \frac{1}{b^{j_{2}-i_{2}+1}}\right) \\
=\frac{b^{3}-5 b^{2}+5 b-1}{6 b^{2}}(m-1)+O(1),
\end{array}
$$

by noticing that the terms for which $i_{1} \notin\left\{i_{2}, j_{2}\right\}$ are all zero. Adding (44), (6) and (7), we obtain the desired formula for $\operatorname{Var}(S(N))$.

\section{Proofs of Theorem 1 and Theorem 2}

Let $N$ be a random variable again, uniformly distributed in $\left\{0,1, \ldots, b^{m}-1\right\}$. Proposition 6 expresses $S(N)$ in terms of independent random variables $a_{1}, \ldots, a_{m}$. In this Section we prove a general central limit theorem and a large deviation result for random variables expressed in terms of independent variables in a similar way. These general results fit into the subject of weakly dependent random variables. The proof of Theorem 9 below is the generalization of the proof in Section 1.3 of [1].

For positive integers $a$ and $m$ let $[m]$ denote the set $\{1,2, \ldots, m\}$, and let

$$
\left(\begin{array}{c}
{[m]} \\
\leq a
\end{array}\right)=\{A \subseteq[m]:|A| \leq a\} .
$$

For a finite set $A$ of integers let $\operatorname{diam} A=\max A-\min A$, and for random variables $X_{1}, \ldots, X_{m}$ let $X_{A}=\left(X_{i}: i \in A\right)$ for any $A \subseteq[m]$.

We are going to use the fact that for any real numbers $\lambda$ and $x$ we have

$$
\begin{aligned}
\Phi(\lambda+x) & =\Phi(\lambda)+O(|x|), \\
\Phi(\lambda(1+x)) & =\Phi(\lambda)+O(|x|) .
\end{aligned}
$$

Note that $\Phi(\lambda+x)-\Phi(\lambda)$ is the integral of $\frac{1}{\sqrt{2 \pi}} e^{-\frac{t^{2}}{2}}$ over an interval of length $|x|$, therefore (8) in fact holds with implied constant $\frac{1}{\sqrt{2 \pi}}$. Since $0 \leq \Phi \leq 1$, (9) holds for any $|x|>\frac{1}{2}$ with implied constant 2. If $|x| \leq \frac{1}{2}$, then for $\lambda \geq 0$ $\Phi(\lambda(1+x))-\Phi(\lambda)$ is an integral over an interval of length $|\lambda x|$, moreover this interval is contained in $[\lambda / 2,3 \lambda / 2]$, therefore the integrand is at most $\frac{1}{\sqrt{2 \pi}} e^{-\frac{\lambda^{2}}{8}}$. Hence 


$$
|\Phi(\lambda(1+x))-\Phi(\lambda)| \leq|\lambda x| \frac{1}{\sqrt{2 \pi}} e^{-\frac{\lambda^{2}}{8}}
$$

and clearly the same is true for $\lambda<0$. Note that $\frac{|\lambda|}{\sqrt{2 \pi}} e^{-\frac{\lambda^{2}}{8}}$ is bounded on $\mathbb{R}$, in fact the maximum is attained at $\lambda= \pm 2$ with maximum value less than 2 . Thus altogether (9) holds with implied constant 2.

Proposition 8. Let $2 \leq a \leq m$ be integers, and let $X_{1}, X_{2}, \ldots, X_{m}$ be independent real valued random variables. For every $A \in\left(\begin{array}{c}{[m]} \\ \leq a\end{array}\right)$ let $f_{A}: \mathbb{R}^{|A|} \rightarrow \mathbb{R}$ be Borel measurable. Suppose that for every $A \in\left(\begin{array}{c}{[m]} \\ \leq a\end{array}\right)$ we have

(i) $\mathrm{E} f_{A}\left(X_{A}\right)=0$,

(ii) $\left|f_{A}\left(X_{A}\right)\right| \leq e^{-c \cdot \operatorname{diam} A}$

for some constant $c>0$. Let $q=\left(\frac{2}{1-e^{-c}}\right)^{a+\frac{1}{2}}$ and $g(x)=\sum_{k=0}^{\infty} \frac{x^{2 a k}}{(2 a k) !}$.

(1) For any integer $k \geq 1$ we have

$$
\mathrm{E}\left(\sum_{A \in\left(\begin{array}{c}
{[m]} \\
\leq a
\end{array}\right)} f_{A}\left(X_{A}\right)\right)^{2 k} \leq q^{2 k}(2 a k) ! \cdot m^{k}
$$

(2) For any real number $\lambda \geq 1$ we have

$$
\operatorname{Pr}\left(\left|\sum_{A \in\left(\begin{array}{c}
{[m]} \\
\leq a
\end{array}\right)} f_{A}\left(X_{A}\right)\right| \geq \lambda q \sqrt{m}\right) \leq \frac{\sqrt[a]{\lambda}}{g(\sqrt[a]{\lambda}-1)}
$$

Proof. (1) Let $L$ denote the left hand side of the claim. By expanding we get

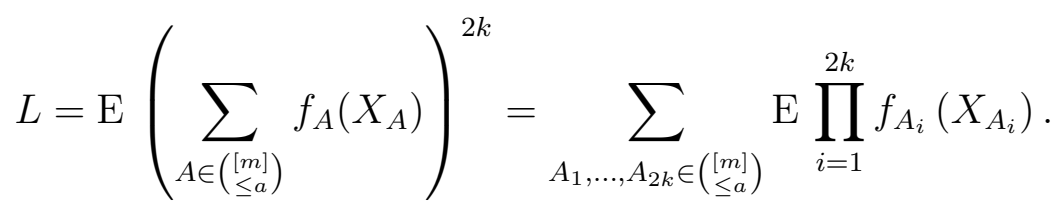

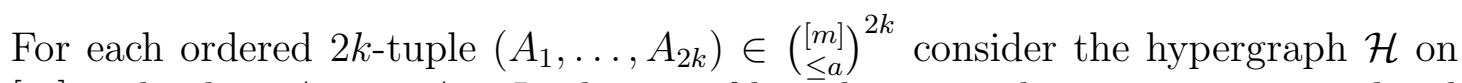
$[m]$ with edges $A_{1}, \ldots, A_{2 k}$. In this proof by a hypergraph we mean an unordered collection of subsets of $[m]$, called edges, with possible repetitions. Let $p$ denote the number of connected components of $\mathcal{H}$, where $1 \leq p \leq 2 k$. Note that if 
$p>k$, then there exists an isolated edge in $\mathcal{H}$, which using the independence of $X_{1}, \ldots, X_{m}$ and condition (i) implies that

$$
\mathrm{E} \prod_{i=1}^{2 k} f_{A_{i}}\left(X_{A_{i}}\right)=0
$$

Suppose now that $1 \leq p \leq k$. Let $\mathcal{C}_{1}, \ldots, \mathcal{C}_{p}$ be the connected components of $\mathcal{H}$, and let $d_{j}=\operatorname{diam} \cup \mathcal{C}_{j}$. The main observation is that the connectedness implies

$$
\begin{gathered}
\operatorname{diam} \bigcup \mathcal{C}_{j} \leq \sum_{A \in \mathcal{C}_{j}} \operatorname{diam} A, \\
\sum_{j=1}^{p} d_{j} \leq \sum_{i=1}^{2 k} \operatorname{diam} A_{i}, \\
\left|\prod_{i=1}^{2 k} f_{A_{i}}\left(X_{A_{i}}\right)\right| \leq \exp \left(-c \cdot \sum_{i=1}^{2 k} \operatorname{diam} A_{i}\right) \leq \exp \left(-c \cdot \sum_{j=1}^{p} d_{j}\right) .
\end{gathered}
$$

Let $M_{j}=\min \bigcup \mathcal{C}_{j}$. Then $\bigcup \mathcal{C}_{j} \subseteq\left[M_{j}, M_{j}+d_{j}\right]$. We are going to group the terms of (10) according to the values $p, M_{1}, \ldots, M_{p}, d_{1}, \ldots, d_{p}$ associated with the corresponding hypergraph $\mathcal{H}$. For given $p, M_{1}, \ldots, M_{p}, d_{1}, \ldots, d_{p}$ all the sets $A_{1}, \ldots, A_{2 k}$ have to be a subset of the set

$$
\bigcup_{j=1}^{p}\left[M_{j}, M_{j}+d_{j}\right]
$$

of size at most $\sum_{j=1}^{p} d_{j}+p$. The number of ordered $2 k$-tuples $\left(A_{1}, \ldots, A_{2 k}\right) \in\left(\begin{array}{c}{[m]} \\ \leq a\end{array}\right){ }^{2 k}$ for which the corresponding hypergraph $\mathcal{H}$ has associated values $p, M_{1}, \ldots, M_{p}$, $d_{1}, \ldots, d_{p}$ is therefore at most

$$
\left(\sum_{j=1}^{p} d_{j}+p\right)^{2 a k}
$$

This together with (11) implies that in (10) we have

$$
L \leq \sum_{p=1}^{k} \sum_{M_{1}, \ldots, M_{p}=1}^{m} \sum_{d_{1}, \ldots, d_{p}=0}^{\infty}\left(\sum_{j=1}^{p} d_{j}+p\right)^{2 a k} \exp \left(-c \sum_{j=1}^{p} d_{j}\right)
$$

Let $d=\sum_{j=1}^{p} d_{j}$. It is known that the number of representations of a given nonnegative integer $d$ in this form is $\left(\begin{array}{c}d+p-1 \\ p-1\end{array}\right)$, therefore we get 


$$
L \leq \sum_{p=1}^{k} \sum_{d=0}^{\infty}\left(\begin{array}{c}
d+p-1 \\
p-1
\end{array}\right)(d+p)^{2 a k} e^{-c d} m^{p} \leq \sum_{p=1}^{k} \sum_{d=0}^{\infty} \frac{\prod_{j=1}^{2 a k+p-1}(d+j)}{(p-1) !} e^{-c d} m^{p} .
$$

The series over $d$ is in fact the well-known Taylor series

$$
\sum_{d=0}^{\infty}(d+\ell) \cdots(d+2)(d+1) x^{d}=\frac{\ell !}{(1-x)^{\ell+1}}
$$

with $\ell=2 a k+p-1$ and $x=e^{-c}$, thus we have

$L \leq \sum_{p=1}^{k} \frac{(2 a k+p-1) !}{(p-1) !} \cdot \frac{m^{p}}{\left(1-e^{-c}\right)^{2 a k+p}}=\sum_{p=1}^{k}\left(\begin{array}{c}2 a k+p-1 \\ 2 a k\end{array}\right)(2 a k) ! \frac{m^{p}}{\left(1-e^{-c}\right)^{2 a k+p}}$.

Here for every $1 \leq p \leq k$ we have

$$
\frac{m^{p}}{\left(1-e^{-c}\right)^{2 a k+p}} \leq \frac{m^{k}}{\left(1-e^{-c}\right)^{(2 a+1) k}} .
$$

We can also use the combinatorial identity and trivial estimate

$$
\left(\begin{array}{l}
n \\
n
\end{array}\right)+\left(\begin{array}{c}
n+1 \\
n
\end{array}\right)+\cdots+\left(\begin{array}{c}
n+k-1 \\
n
\end{array}\right)=\left(\begin{array}{c}
n+k \\
n+1
\end{array}\right) \leq 2^{n+k}
$$

with $n=2 a k$ to finally obtain

$$
L \leq 2^{(2 a+1) k}(2 a k) ! \frac{m^{k}}{\left(1-e^{-c}\right)^{(2 a+1) k}}=q^{2 k}(2 a k) ! m^{k} .
$$

(2) Let $P$ denote the probability in the claim. Note that $g(x)$ is monotone increasing on $[0, \infty)$. Therefore for any real number $0<\alpha<1$ we have

$$
\begin{aligned}
& P=\operatorname{Pr}\left(\left|\sum_{A \in\left(\begin{array}{c}
{[m]} \\
\leq a
\end{array}\right)} f_{A}\left(X_{A}\right)\right| \geq \lambda q \sqrt{m}\right) \\
& =\operatorname{Pr}\left(g\left(\frac{\alpha}{q^{\frac{1}{a}} m^{\frac{1}{2 a}}}\left|\sum_{A \in\left(\begin{array}{c}
{[m]} \\
\leq a
\end{array}\right)} f_{A}\left(X_{A}\right)\right|^{\frac{1}{a}}\right) \geq g\left(\alpha \lambda^{\frac{1}{a}}\right)\right) .
\end{aligned}
$$

Applying Markov's inequality and Lebesgue's monotone convergence theorem we obtain that 


$$
P \leq \frac{1}{g\left(\alpha \lambda^{\frac{1}{a}}\right)} \sum_{k=0}^{\infty} \frac{\alpha^{2 a k}}{q^{2 k} m^{k}(2 a k) !} \mathrm{E}\left(\sum_{A \in\left(\begin{array}{c}
{[m]} \\
\leq a
\end{array}\right)} f_{A}\left(X_{A}\right)\right)^{2 k}
$$

Proposition 8 (1) yields the upper bound

$$
P \leq \frac{1}{g\left(\alpha \lambda^{\frac{1}{a}}\right)} \sum_{k=0}^{\infty} \alpha^{2 a k}=\frac{1}{1-\alpha^{2 a}} \cdot \frac{1}{g\left(\alpha \lambda^{\frac{1}{a}}\right)} .
$$

Choosing $\alpha=1-\lambda^{-\frac{1}{a}}$ and noticing $1-\alpha^{2 a} \geq 1-\alpha=\lambda^{-\frac{1}{a}}$ finishes the proof.

Theorem 9. Let $2 \leq a \leq m$ be integers, and let $X_{1}, X_{2}, \ldots, X_{m}$ be independent real valued random variables. For every $A \in\left(\begin{array}{c}{[m]} \\ \leq a\end{array}\right)$ let $f_{A}: \mathbb{R}^{|A|} \rightarrow \mathbb{R}$ be Borel measurable. Suppose that for every $A \in\left(\begin{array}{c}{[m]} \\ \leq a\end{array}\right)$ we have

(i) $\mathrm{E} f_{A}\left(X_{A}\right)=0$,

(ii) $\left|f_{A}\left(X_{A}\right)\right| \leq e^{-c \cdot \operatorname{diam} A}$,

(iii) $\sigma_{m}^{2}=\mathrm{E}\left(\sum_{A \in\left(\begin{array}{c}{[m]} \\ \leq a\end{array}\right)} f_{A}\left(X_{A}\right)\right)^{2}>0$

for some constant $c>0$. Then for any real number $\lambda$ we have

$$
\operatorname{Pr}\left(\frac{1}{\sigma_{m}} \sum_{A \in\left(\begin{array}{c}
{[m]} \\
\leq a
\end{array}\right)} f_{A}\left(X_{A}\right)<\lambda\right)=\Phi(\lambda)+O\left(\sqrt[4]{\log m} \cdot \frac{m^{\frac{3}{4}}}{\sigma_{m}^{2}}\right) .
$$

The implied constant in the error term depends only on a and $c$.

Note that Proposition 8 (1) with $k=1$ implies that $\sigma_{m}^{2}=O(m)$. The smallest attainable error term in Theorem 9 is therefore $O\left(\frac{\sqrt[4]{\log m}}{\sqrt[4]{m}}\right)$, which holds whenever $\sigma_{m}^{2}>d \cdot m$ for some constant $d>0$.

Proof. Throughout this proof the implied constants in the $O$ notation will depend only on $a$ and $c$. We may assume $\sigma_{m}^{2} \geq m^{\frac{3}{4}}$, otherwise the error term is larger than 1. We start by partitioning the set $[m]$ into $m_{0}$ intervals of integers $I_{1}, I_{2}, \ldots, I_{m_{0}}$, in such a way that $\max I_{i}=\min I_{i+1}-1$ and $\left|I_{i}\right|=\Theta\left(\frac{m}{m_{0}}\right)$ for any $i$. Assume $\left|I_{i}\right|>\frac{6}{c} \log m$ for all $i$. Let 


$$
\begin{aligned}
& Y_{i}=\sum_{\substack{A \in\left(\begin{array}{c}
I_{i} \\
\leq a
\end{array}\right) \\
f_{A}}}\left(X_{A}\right),
\end{aligned}
$$

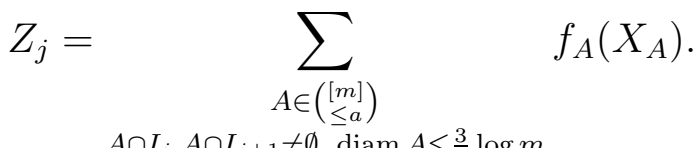

$$
\begin{aligned}
& A \cap I_{j}, A \cap I_{j+1} \neq \emptyset \text {, diam } A \leq \frac{3}{c} \log m
\end{aligned}
$$

Then the random variable we are interested in can be written as

$$
\sum_{A \in\left(\begin{array}{c}
{[m]} \\
\leq a
\end{array}\right)} f_{A}\left(X_{A}\right)=\sum_{i=1}^{m_{0}} Y_{i}+\sum_{j=1}^{m_{0}-1} Z_{j}+W
$$

where the random variable $W$ is defined by (12). Then $Y_{1}, \ldots, Y_{m_{0}}$ are independent, and the assumption $\left|I_{i}\right|>\frac{6}{c} \log m$ implies that $Z_{1}, \ldots, Z_{m_{0}-1}$ are also independent.

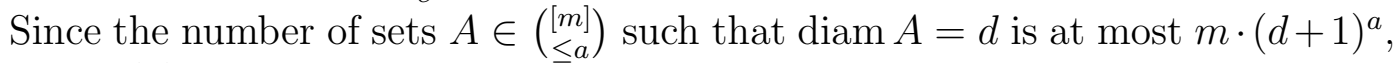
condition (ii) implies that

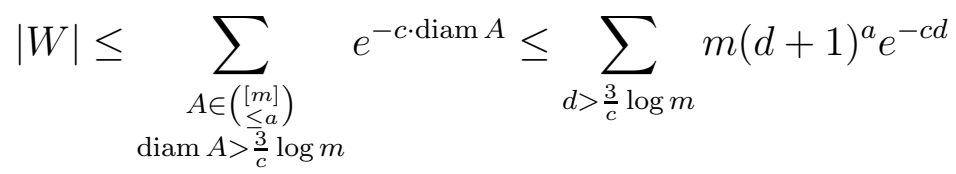

$$
\begin{aligned}
& =O\left(m \log ^{a} m \cdot e^{-c \frac{3}{c} \log m}\right)=O\left(\frac{1}{m}\right) \text {. }
\end{aligned}
$$

Similarly,

$$
\left|Y_{i}\right| \leq \sum_{A \in\left(\begin{array}{c}
I_{i} \\
\leq a
\end{array}\right)} e^{-c \cdot \operatorname{diam} A} \leq \sum_{d=0}^{\infty}\left|I_{i}\right|(d+1)^{a} e^{-c d}=O\left(\left|I_{i}\right|\right)=O\left(\frac{m}{m_{0}}\right)
$$

The number of sets $A \in\left(\begin{array}{c}{[m]} \\ \leq a\end{array}\right)$ with $A \subseteq\left[\max I_{j}-d\right.$, $\left.\max I_{j}+d\right]$ is at most $(2 d+1)^{a}$, therefore condition (ii) implies

$$
\left|Z_{j}\right| \leq \sum_{d=0}^{\infty}(2 d+1)^{a} e^{-c d}=O(1)
$$

Finally, note that the number of sets $A \in\left(\begin{array}{c}{\left[\begin{array}{c}m \\ <a\end{array}\right)} \\ )\end{array}\right)$ such that $\operatorname{diam} A=d_{1}$ which intersect $\left[\max I_{j}-d_{2}, \max I_{j}+d_{2}\right]$ is at most $\left(2 d_{1}+2 d_{2}+1\right)^{a}$, thus from conditions (i) and (ii) we obtain that for any $i$ and $j$ we have 


$$
\left|\mathrm{E}\left(Y_{i} Z_{j}\right)\right| \leq \sum_{d_{1}, d_{2} \geq 0}\left(2 d_{1}+2 d_{2}+1\right)^{a}\left(2 d_{2}+1\right)^{a} e^{-c d_{1}} e^{-c d_{2}}=O(1) .
$$

By taking the variance of (12) we get

$$
\begin{aligned}
\sigma_{m}^{2}=\sum_{i=1}^{m_{0}} \operatorname{Var}\left(Y_{i}\right)+\sum_{j=1}^{m_{0}-1} \operatorname{Var}\left(Z_{j}\right) & +2 \sum_{i=1}^{m_{0}} \sum_{j=1}^{m_{0}-1} \mathrm{E}\left(Y_{i} Z_{j}\right) \\
& +2 \sum_{i=1}^{m_{0}} \mathrm{E}\left(Y_{i} W\right)+2 \sum_{j=1}^{m_{0}-1} \mathrm{E}\left(Z_{j} W\right)+\operatorname{Var}(W) .
\end{aligned}
$$

By noticing that $\mathrm{E}\left(Y_{i} Z_{j}\right)=0$ unless $i=j$ or $i=j+1$, the bounds (13) $-(16)$ imply

$$
\sigma_{m}^{2}=\sum_{i=1}^{m_{0}} \operatorname{Var}\left(Y_{i}\right)+O\left(m_{0}\right) .
$$

We now want to apply the Berry-Esseen theorem to the sum $\sum_{i=1}^{m_{0}} Y_{i}$ of independent random variables. Applying Proposition 8 (1) with $k=2$ we obtain

$$
\mathrm{E} Y_{i}^{4}=O\left(\left|I_{i}\right|^{2}\right)=O\left(\frac{m^{2}}{m_{0}^{2}}\right)
$$

therefore the Hölder inequality implies

$$
\sum_{i=1}^{m_{0}} \mathrm{E}\left|Y_{i}\right|^{3} \leq \sum_{i=1}^{m_{0}}\left(\mathrm{E} Y_{i}^{4}\right)^{\frac{3}{4}}=O\left(\frac{m^{\frac{3}{2}}}{\sqrt{m_{0}}}\right) .
$$

As long as $m_{0}=o\left(\sigma_{m}^{2}\right)$, we can see from (17) that

$$
\left(\sum_{i=1}^{m_{0}} \operatorname{Var}\left(Y_{i}\right)\right)^{\frac{3}{2}}=\sigma_{m}^{3}(1+o(1)) .
$$

Therefore the Berry-Esseen theorem ([2] Section 9.1 Theorem 3) implies that

$$
\begin{aligned}
\operatorname{Pr}\left(\frac{1}{\sqrt{\sum_{i=1}^{m_{0}} \operatorname{Var}\left(Y_{i}\right)}} \sum_{i=1}^{m_{0}} Y_{i}<\lambda\right) & =\Phi(\lambda)+O\left(\frac{\sum_{i=1}^{m_{0}} \mathrm{E}\left|Y_{i}\right|^{3}}{\left(\sum_{i=1}^{m_{0}} \operatorname{Var}\left(Y_{i}\right)\right)^{\frac{3}{2}}}\right) \\
& =\Phi(\lambda)+O\left(\frac{m^{\frac{3}{2}}}{\sigma_{m}^{3} \sqrt{m_{0}}}\right) .
\end{aligned}
$$


From (17) we obtain

$$
\frac{1}{\sqrt{\sum_{i=1}^{m_{0}} \operatorname{Var}\left(Y_{i}\right)}}=\frac{1}{\sigma_{m}} \cdot\left(1+O\left(\frac{m_{0}}{\sigma_{m}^{2}}\right)\right) .
$$

Therefore we can use (9) with $x=O\left(\frac{m_{0}}{\sigma_{m}^{2}}\right)$ to replace the normalizing factor in the probability in (18) by $\frac{1}{\sigma_{m}}$ to get

$$
\operatorname{Pr}\left(\frac{1}{\sigma_{m}} \sum_{i=1}^{m_{0}} Y_{i}<\lambda\right)=\Phi(\lambda)+O\left(\frac{m^{\frac{3}{2}}}{\sigma_{m}^{3} \sqrt{m_{0}}}+\frac{m_{0}}{\sigma_{m}^{2}}\right) .
$$

Recall that a simple version of the Chernoff bound states that if $\zeta_{1}, \cdots, \zeta_{n}$ are independent random variables such that $\mathrm{E}\left(\zeta_{j}\right)=0$ and $\left|\zeta_{j}\right| \leq 1$ for every $1 \leq j \leq n$, then for any $t>0$ we have

$$
\operatorname{Pr}\left(\left|\sum_{j=1}^{n} \zeta_{j}\right|>t \sqrt{n}\right) \leq 2 e^{-\frac{t^{2}}{2}} .
$$

According to (15) there exists a constant $K>0$ such that $\left|Z_{j}\right| \leq K$ for all $j$. Condition (i) ensures that $\mathrm{E}\left(Z_{j}\right)=0$ for all $j$. Therefore we can apply the Chernoff bound to $\zeta_{j}=Z_{j} / K$ with $n=m_{0}-1$ and $t=\sqrt{\log m}$ to obtain

$$
\operatorname{Pr}\left(\frac{1}{\sigma_{m}}\left|\sum_{j=1}^{m_{0}-1} Z_{j}\right|>K \sqrt{\log m} \frac{\sqrt{m_{0}-1}}{\sigma_{m}}\right) \leq \frac{2}{\sqrt{m}} .
$$

From (12), (13) and (20) we get

$$
\begin{aligned}
& \operatorname{Pr}\left(\frac{1}{\sigma_{m}} \sum_{A \in\left(\begin{array}{c}
{[m]} \\
\leq a
\end{array}\right)} f_{A}\left(X_{A}\right)<\lambda\right) \\
& =\operatorname{Pr}\left(\frac{1}{\sigma_{m}} \sum_{i=1}^{m_{0}} Y_{i}<\lambda+O\left(\sqrt{\log m} \frac{\sqrt{m_{0}}}{\sigma_{m}}+\frac{1}{\sigma_{m} m}\right)\right)+O\left(\frac{1}{\sqrt{m}}\right) .
\end{aligned}
$$

Combining (19) and (18) with $x=O\left(\sqrt{\log m} \frac{\sqrt{m_{0}}}{\sigma_{m}}+\frac{1}{\sigma_{m} m}\right)$ we finally obtain 


$$
\begin{aligned}
\operatorname{Pr}\left(\frac{1}{\sigma_{m}} \sum_{A \in\left(\begin{array}{c}
{[m]} \\
\leq a
\end{array}\right)} f_{A}\left(X_{A}\right)<\lambda\right) \\
\quad=\Phi(\lambda)+O\left(\frac{m^{\frac{3}{2}}}{\sigma_{m}^{3} \sqrt{m_{0}}}+\frac{m_{0}}{\sigma_{m}^{2}}+\sqrt{\log m} \frac{\sqrt{m_{0}}}{\sigma_{m}}+\frac{1}{\sigma_{m} m}+\frac{1}{\sqrt{m}}\right) .
\end{aligned}
$$

The optimal choice for $m_{0}$ is when the first and the third error terms are equal, which holds when

$$
m_{0}=\Theta\left(\frac{m^{\frac{3}{2}}}{\sqrt{\log m} \cdot \sigma_{m}^{2}}\right) .
$$

Using $\sigma_{m}^{2} \geq m^{\frac{3}{4}}$ it is easy to check that for this choice of $m_{0}$ both our assumptions $\left|I_{i}\right|>\frac{6 \log m}{c}$ and $m_{0}=o\left(\sigma_{m}^{2}\right)$ hold.

Proof of Theorem 1. First, suppose that $M=b^{m}$ for some integer $m \geq 2$. Let $N$ be a random variable uniformly distributed in $\left\{0,1, \ldots, b^{m}-1\right\}$. Then the base $b$ digits $a_{1}, \ldots, a_{m}$ of $N$ are independent random variables. Let $K>0$ be a constant for which

$$
\begin{gathered}
\left|\frac{(b+1) a_{i}-a_{i}^{2}}{2 b}-\mathrm{E} \frac{(b+1) a_{i}-a_{i}^{2}}{2 b}\right| \leq K b, \\
\left|\frac{a_{i} a_{j}}{b}-\mathrm{E}\left(\frac{a_{i} a_{j}}{b}\right)\right| \leq K b
\end{gathered}
$$

for any $1 \leq i<j \leq m$. Using Proposition 6 we can write $S(N)$ in the form

$$
S(N)-\mathrm{E}(S(N))=K b \sum_{A \in\left(\begin{array}{c}
{[m]} \\
\leq 2
\end{array}\right)} f_{A}\left(a_{A}\right),
$$

where $f_{\emptyset}=0, f_{\{i\}}(x)=\frac{(b+1) x-x^{2}}{2 K b^{2}}-\mathrm{E} \frac{(b+1) a_{i}-a_{i}^{2}}{2 K b^{2}}$ and for $1 \leq i<j \leq m$

$$
f_{\{i, j\}}(x, y)=-\left(\frac{x y}{K b^{2}}-\mathrm{E}\left(\frac{a_{i} a_{j}}{K b^{2}}\right)\right) \cdot \frac{1}{b^{j-i}} .
$$

Then the conditions of Theorem 9 are satisfied with $a=2$ and $c=\log 2$. According to Proposition 7 we have $\sigma_{m}^{2}=\frac{1}{K^{2} b^{2}} \operatorname{Var}(S(N))=\Theta(m)$, hence we obtain

$$
\operatorname{Pr}\left(\frac{S(N)-\mathrm{E}(S(N))}{\sqrt{\operatorname{Var}(S(N))}}<\lambda\right)=\Phi(\lambda)+O\left(\frac{\sqrt[4]{\log m}}{\sqrt[4]{m}}\right) .
$$


Since $d(b)=\Theta\left(b^{2}\right)$, from Proposition 7 we can see that

$$
\begin{aligned}
\frac{1}{\sqrt{\operatorname{Var}(S(N))}}=\frac{1}{\sqrt{d(b) m}}\left(1+O\left(\frac{1}{b m}\right)\right), \\
\frac{\mathrm{E}(S(N))}{\sqrt{d(b) m}}=\frac{c(b) m}{\sqrt{d(b) m}}+O\left(\frac{1}{b \sqrt{m}}\right) .
\end{aligned}
$$

Hence if we replace $\operatorname{Var}(S(N))$ by $d(b) m$, and then $\mathrm{E}(S(N))$ by $c(b) m$ in the probability, then using (9) with $x=O\left(\frac{1}{b m}\right)$ and (8) with $x=O\left(\frac{1}{b \sqrt{m}}\right)$ the error we make is $O\left(\frac{1}{b m}+\frac{1}{b \sqrt{m}}\right)$. Thus

$$
\operatorname{Pr}\left(\frac{S(N)-c(b) m}{\sqrt{d(b) m}}<\lambda\right)=\Phi(\lambda)+O\left(\frac{\sqrt[4]{\log m}}{\sqrt[4]{m}}\right) .
$$

We now show that (21) holds for any $M>b^{2}$. Let $M=\sum_{i=1}^{m} c_{i} b^{i-1}$ be the base $b$ representation of $M$, where $c_{i} \in\{0,1, \ldots, b-1\}$ and $c_{m}>0$. Let

$$
M^{*}=\sum_{m-\log m-1 \leq i \leq m} c_{i} b^{i-1}
$$

Let $N$ be a random variable uniformly distributed in $\left\{0,1, \ldots, M^{*}-1\right\}$, and consider its base $b$ representation $N=\sum_{i=1}^{m} a_{i} b^{i-1}$. Note that we allow $a_{m}$ to be zero. Then the random variables $\left(a_{i}: 1 \leq i<m-\log m-1\right)$ are independent, and each is uniformly distributed in $\{0,1, \ldots, b-1\}$. Let us introduce new random variables $a_{j}^{*}$ for every $m-\log m-1 \leq j \leq m$, such that

$$
\left(a_{i}, a_{j}^{*}: 1 \leq i<m-\log m-1 \leq j \leq m\right)
$$

are identically distributed independent random variables. Let

$$
N^{*}=\sum_{1 \leq i<m-\log m-1} a_{i} b^{i-1}+\sum_{m-\log m-1 \leq j \leq m} a_{j}^{*} b^{j-1} .
$$

Then $S\left(N^{*}\right)$ satisfies (21). Note that there are $O(\log m)$ base $b$ digits at which $N$ and $N^{*}$ differ. According to the formula in Proposition 6, if a single base $b$ digit of $N$ is changed, $S(N)$ can change by at most $O(b)$. Hence $S\left(N^{*}\right)=$ $S(N)+O(b \log m)$. Using (18) with $x=O\left(\frac{\log m}{\sqrt{m}}\right)$, the error of replacing $S\left(N^{*}\right)$ in (21) by $S(N)$ is $O\left(\frac{\log m}{\sqrt{m}}\right)$, therefore

$$
\frac{1}{M^{*}}\left|\left\{0 \leq N<M^{*}: \frac{S(N)-c(b) m}{\sqrt{d(b) m}}<\lambda\right\}\right|=\Phi(\lambda)+O\left(\frac{\sqrt[4]{\log m}}{\sqrt[4]{m}}\right) .
$$


Here the error of replacing $M^{*}$ by $M$ is

$$
O\left(\frac{M-M^{*}}{M}\right)=O\left(\frac{b^{m-\log m-1}}{b^{m-1}}\right)=O\left(\frac{\sqrt[4]{\log m}}{\sqrt[4]{m}}\right) .
$$

Finally, note that $\frac{M}{m} \leq N \leq M$ with probability $1-O\left(\frac{1}{m}\right)$, and for all such $N$ we have $\log _{b} N=m+O(\log m)$. Using (8) with $x=O\left(\frac{\log m}{\sqrt{m}}\right)$, the error of replacing $c(b) m$ by $c(b) \log _{b} N$ is $O\left(\frac{\log m}{\sqrt{m}}\right)$. Using (9) with $x=O\left(\frac{\log m}{m}\right)$, the error of replacing $\sqrt{d(b) m}$ by $\sqrt{d(b) \log _{b} N}$ is $O\left(\frac{\log m}{m}\right)$. Hence we get

$$
\frac{1}{M}\left|\left\{0 \leq N<M: \frac{S(N)-c(b) \log _{b} N}{\sqrt{d(b) \log _{b} N}}<\lambda\right\}\right|=\Phi(\lambda)+O\left(\frac{\sqrt[4]{\log m}}{\sqrt[4]{m}}\right) .
$$

The error term can be expressed in terms of $M$ by noting $m \geq \log _{b} M$.

Proof of Theorem 2. First, assume $M=b^{m}$ for some integer $m \geq 2$. Let $N$ be a random variable uniformly distributed in $\left\{0,1, \ldots, b^{m}-1\right\}$, and let $N=$ $\sum_{i=1}^{m} a_{i} b^{i-1}$ be the base $b$ representation of $N$, where $a_{1}, \ldots, a_{m}$ are independent random variables, each uniformly distributed in $\{0,1, \ldots, b-1\}$. Note that for any $1 \leq i<j \leq m$ we have

$$
\begin{gathered}
\left|\frac{(b+1) a_{i}-a_{i}^{2}}{2 b}-\frac{(b+1) \mathrm{E}\left(a_{i}\right)-\mathrm{E}\left(a_{i}^{2}\right)}{2 b}\right| \leq \frac{3}{4} b, \\
\left|\frac{a_{i} a_{j}}{b}-\frac{\mathrm{E}\left(a_{i}\right) \mathrm{E}\left(a_{j}\right)}{b}\right| \leq \frac{3}{4} b .
\end{gathered}
$$

Using Proposition 6 we can write $S(N)$ in the form

$$
S(N)-\mathrm{E}(S(N))=\frac{3}{4} b \sum_{A \in\left(\begin{array}{l}
{[m]} \\
\leq 2
\end{array}\right)} f_{A}\left(a_{A}\right),
$$

where $f_{\emptyset}=0, f_{\{i\}}(x)=\frac{4}{3 b} \frac{(b+1) x-x^{2}}{2 b}-\frac{4}{3 b} \mathrm{E} \frac{(b+1) a_{i}-a_{i}^{2}}{2 b}$ and for $1 \leq i<j \leq m$

$$
f_{\{i, j\}}(x, y)=-\frac{4}{3 b}\left(\frac{x y}{b}-\mathrm{E}\left(\frac{a_{i} a_{j}}{b}\right)\right) \cdot \frac{1}{b^{j-i}} .
$$

Then the conditions of Proposition 8 (2) are satisfied with $a=2, c=\log 2, q=32$ and

$$
g(x)=\sum_{k=0}^{\infty} \frac{x^{4 k}}{(4 k) !}=\frac{e^{x}+e^{-x}}{4}+\frac{\cos x}{2} \geq \frac{e^{x}-2}{4} .
$$


Therefore Proposition 8 (2) yields

$$
\begin{aligned}
& \operatorname{Pr}(|S(N)-\mathrm{E}(S(N))| \geq 24 \lambda b \sqrt{m})=\operatorname{Pr}\left(\left|\sum_{A \in\left(\begin{array}{c}
{[m]} \\
\leq 2
\end{array}\right)} f_{A}\left(a_{A}\right)\right| \geq 32 \lambda \sqrt{m}\right) \\
& \leq \frac{4 \sqrt{\lambda}}{e^{\sqrt{\lambda}-1}-2} \text {. }
\end{aligned}
$$

Now we prove (22) holds for any integer $M>b$. Let $M=\sum_{i=1}^{m} c_{i} b^{i-1}$ be the base $b$ representation of $M$, where $c_{i} \in\{0,1, \ldots, b-1\}$ and $c_{m}>0$. Let

$$
M^{*}=\sum_{m-\sqrt{m}+1 \leq i \leq m} c_{i} b^{i-1} .
$$

Let $N$ be a random variable uniformly distributed in $\left\{0,1, \ldots, M^{*}-1\right\}$, and consider its base $b$ representation $N=\sum_{i=1}^{m} a_{i} b^{i-1}$. Then $\left(a_{i}: 1 \leq i<m-\sqrt{m}+1\right)$ are independent random variables, each uniformly distributed in $\{0,1, \ldots, b-1\}$. Let us introduce new random variables $a_{j}^{*}$ for $m-\sqrt{m}+1 \leq j \leq m$ such that

$$
\left(a_{i}, a_{j}^{*}: 1 \leq i<m-\sqrt{m}+1 \leq j \leq m\right)
$$

are identically distributed independent random variables. Let

$$
N^{*}=\sum_{1 \leq i<m-\sqrt{m}+1} a_{i} b^{i-1}+\sum_{m-\sqrt{m}+1 \leq j \leq m} a_{j}^{*} b^{j-1} .
$$

Then $S\left(N^{*}\right)$ satisfies (22). Using Proposition [6] and Proposition 7 we get the following estimates:

$$
\begin{aligned}
\left|\mathrm{E}\left(S\left(N^{*}\right)\right)-\frac{b^{2}-1}{12 b} m\right| & \leq \frac{1}{4} \leq \frac{\lambda b \sqrt{m}}{24 \sqrt{2}} \\
\left|\frac{b^{2}-1}{12 b} m-\frac{b^{2}-1}{12 b} \log _{b} M\right| & \leq \frac{b^{2}-1}{12 b} \leq \frac{\lambda b \sqrt{m}}{36 \sqrt{2}} \\
\left|S(N)-S\left(N^{*}\right)\right| & \leq \frac{(b+1)^{2}}{8 b} \sqrt{m}+2 \sqrt{m} \leq \frac{41}{96} \lambda b \sqrt{m} .
\end{aligned}
$$

Since

$$
24+\frac{1}{24 \sqrt{2}}+\frac{1}{36 \sqrt{2}}+\frac{41}{96}<25
$$

these estimates imply 


$$
\frac{1}{M^{*}}\left|\left\{0 \leq N<M^{*}:\left|S(N)-\frac{b^{2}-1}{12 b} \log _{b} M\right| \geq 25 \lambda b \sqrt{\log _{b} M+1}\right\}\right| \leq \frac{4 \sqrt{\lambda}}{e^{\sqrt{\lambda}-1}-2} .
$$

Finally, note that the error of replacing $M^{*}$ by $M$ is at most

$$
\frac{M-M^{*}}{M} \leq \frac{b^{m-\sqrt{m}+1}}{b^{m-1}} \leq \frac{1}{b^{\sqrt{\log _{b} M}-2}} .
$$

\section{Proofs of Theorem 3 and Theorem 4}

In this Section the proofs of Theorem 3 , Theorem 4 and Proposition 5 are given. We start by estimating an exponential sum in terms of the base $b$ van der Corput sequence. Proposition 10 below is a special case of Lemma 3 in [6]. Nevertheless, for the sake of completeness a proof is included.

Proposition 10. Let $b \geq 2$ be an integer and let $x_{n}$ denote the base $b$ van der Corput sequence. If $\ell$ is an integer such that $b^{s} \nmid \ell$ for some positive integer $s$, then for any positive integer $N$ we have

$$
\left|\sum_{n=0}^{N-1} e^{2 \pi i \ell x_{n}}\right|<b^{s}
$$

Proof. Let $N=\sum_{j=1}^{m} a_{j} b^{j-1}$ be the base $b$ representation of $N$ with base $b$ digits $a_{j} \in\{0,1, \ldots, b-1\}$ with $a_{m} \neq 0$. By splitting the sum we get

$$
\left|\sum_{n=0}^{N-1} e^{2 \pi i \ell x_{n}}\right| \leq\left|\sum_{n=0}^{a_{m} b^{m-1}-1} e^{2 \pi i \ell x_{n}}\right|+\left|\sum_{n=a_{m} b^{m-1}}^{N-1} e^{2 \pi i \ell x_{n}}\right|
$$

Note that for any $a_{m} b^{m-1} \leq n<N$ the base $b$ representation of $n$ starts with the digit $a_{m}$. From the definition of the base $b$ van der Corput sequence we know that for any such $n$ we have $x_{n}=x_{n-a_{m} b^{m-1}}+\frac{a_{m}}{b^{m}}$, therefore we can reindex the second sum to obtain

$$
\left|\sum_{n=a_{m} b^{m-1}}^{N-1} e^{2 \pi i \ell x_{n}}\right|=\left|e^{2 \pi i \ell \frac{a_{m}}{b^{m}}} \sum_{n=0}^{N-a_{m} b^{m-1}-1} e^{2 \pi i \ell x_{n}}\right|
$$

Using the base $b$ representation of $N$, repeated application of the triangle inequality in (23) yields 


$$
\left|\sum_{n=0}^{N-1} e^{2 \pi i \ell x_{n}}\right| \leq \sum_{j=1}^{m}\left|\sum_{n=0}^{a_{j} b^{j-1}-1} e^{2 \pi i \ell x_{n}}\right|
$$

For any $1 \leq j \leq m$ we have

$$
\left\{x_{n}: 0 \leq n<a_{j} b^{j-1}\right\}=\left\{\frac{k}{b^{j-1}}+\frac{a}{b^{j}}: 0 \leq k<b^{j-1}, \quad 0 \leq a<a_{j}\right\},
$$

therefore

$$
\left|\sum_{n=0}^{a_{j} b^{j-1}-1} e^{2 \pi i \ell x_{n}}\right|=\left|\sum_{k=0}^{b^{j-1}-1} e^{2 \pi i \frac{\ell}{b^{j}-1}} k\right| \cdot\left|\sum_{a=0}^{a_{j}-1} e^{2 \pi i \ell \frac{a}{b^{j}}}\right| .
$$

The assumption $b^{s} \nmid \ell$ implies that the first factor is zero whenever $s \leq j-1$. Thus we get from (24) that

$$
\left|\sum_{n=0}^{N-1} e^{2 \pi i \ell x_{n}}\right| \leq \sum_{j=1}^{s}\left|\sum_{n=0}^{a_{j} b^{j-1}-1} e^{2 \pi i \ell x_{n}}\right| \leq \sum_{j=1}^{s} a_{j} b^{j-1}<b^{s} .
$$

Proof of Theorem 3. It is enough to prove the theorem in the special case when $p$ is a positive even integer. Indeed, if $p \geq 1$ is arbitrary, we can choose a positive even integer $p^{\prime}>p$. Observation (11) then implies

$$
S(N) \leq\left\|\Delta_{N}\right\|_{p} \leq\left\|\Delta_{N}\right\|_{p^{\prime}}
$$

Theorem 1 and Theorem 3 with $p^{\prime}$ thus imply Theorem 3 with $p$.

From now on we assume $p$ is a positive even integer. Every implied constant in the $O$ notation will depend only on $p$. From the alternative form of the discrepancy function

$$
\Delta_{N}(x)=\sum_{n=0}^{N-1}\left(\chi_{\left(x_{n}, 1\right]}(x)-x\right)
$$

where $\chi$ denotes the characteristic function of a set, one obtains via routine integration that for any integer $\ell \neq 0$ we have

$$
\int_{0}^{1} \Delta_{N}(x) e^{-2 \pi i \ell x} \mathrm{~d} x=\frac{1}{2 \pi i \ell} \sum_{n=0}^{N-1} e^{-2 \pi i \ell x_{n}} .
$$


Therefore Parseval's formula and observation (11) yield

$$
\int_{0}^{1}\left(\Delta_{N}(x)-S(N)\right)^{2} \mathrm{~d} x=\sum_{\ell \neq 0} \frac{1}{4 \pi^{2} \ell^{2}}\left|\sum_{n=0}^{N-1} e^{-2 \pi i \ell x_{n}}\right|^{2}
$$

Let $N=\sum_{j=1}^{m} a_{j} b^{j-1}$ be the base $b$ representation of $N$, where $a_{j} \in\{0,1, \ldots, b-1\}$ and $a_{m}>0$. Note $N<b^{m}$. Let $b^{s} \| \ell$ denote the fact that $b^{s} \mid \ell$ but $b^{s+1} \nmid \ell$. By splitting the sum according to the highest power of $b$ dividing $\ell$, and applying Proposition 10 and a trivial estimate we obtain

$$
\begin{aligned}
& \int_{0}^{1}\left(\Delta_{N}(x)-S(N)\right)^{2} \mathrm{~d} x \\
& =\sum_{s=0}^{m-2} \sum_{\substack{\ell \neq 0 \\
b^{s} \| \ell}} \frac{1}{4 \pi^{2} \ell^{2}}\left|\sum_{n=0}^{N-1} e^{-2 \pi i \ell x_{n}}\right|^{2}+\sum_{\substack{\ell \neq 0 \\
b^{m-1} \mid \ell}} \frac{1}{4 \pi^{2} \ell^{2}}\left|\sum_{n=0}^{N-1} e^{-2 \pi i \ell x_{n}}\right|^{2} \\
& \leq \sum_{s=0}^{m-2} \sum_{\substack{\ell \neq 0 \\
b^{s} \| \ell}} \frac{1}{4 \pi^{2} \ell^{2}} b^{2 s+2}+\sum_{\substack{\ell \neq 0 \\
b^{m-1} \mid \ell}} \frac{1}{4 \pi^{2} \ell^{2}} b^{2 m} \\
& \leq \sum_{s=0}^{m-1} \sum_{t \neq 0} \frac{b^{2}}{4 \pi^{2} t^{2}}=\frac{b^{2}}{12} m \leq \frac{b^{2}}{12}\left(\log _{b} N+1\right) \text {. }
\end{aligned}
$$

For a positive even integer $p$ consider the binomial formula

$$
\begin{aligned}
\Delta_{N}(x)^{p}=S(N)^{p}+p S(N)^{p-1}\left(\Delta_{N}(x)\right. & -S(N)) \\
& +\sum_{k=2}^{p}\left(\begin{array}{l}
p \\
k
\end{array}\right) S(N)^{p-k}\left(\Delta_{N}(x)-S(N)\right)^{k} .
\end{aligned}
$$

By integrating on $[0,1]$ we get

$$
\int_{0}^{1} \Delta_{N}(x)^{p} \mathrm{~d} x=S(N)^{p}+\sum_{k=2}^{p}\left(\begin{array}{l}
p \\
k
\end{array}\right) S(N)^{p-k} \int_{0}^{1}\left(\Delta_{N}(x)-S(N)\right)^{k} \mathrm{~d} x .
$$

Using the facts that $\Delta_{N}(x)=O\left(b\left(\log _{b} N+1\right)\right)$ and $S(N)=O\left(b\left(\log _{b} N+1\right)\right)$, we get from (25) that for any $2 \leq k \leq p$ 


$$
\begin{aligned}
\int_{0}^{1}\left(\Delta_{N}(x)-S(N)\right)^{k} \mathrm{~d} x & \leq \sup _{x \in[0,1]}\left|\Delta_{N}(x)-S(N)\right|^{k-2} \int_{0}^{1}\left(\Delta_{N}(x)-S(N)\right)^{2} \mathrm{~d} x \\
& =O\left(b^{k}\left(\log _{b} N+1\right)^{k-1}\right) .
\end{aligned}
$$

Thus we have

$$
\int_{0}^{1} \Delta_{N}(x)^{p} \mathrm{~d} x=S(N)^{p}+O\left(b^{p}\left(\log _{b} N+1\right)^{p-1}\right) .
$$

Now we prove the theorem. Let $M>b^{2}$, and let $N$ be a random variable uniformly distributed in $\{0,1, \ldots, M-1\}$. We know from Theorem 1 that the event

has probability

$$
\frac{S(N)-c(b) \log _{b} N}{\sqrt{d(b) \log _{b} N}}>-\frac{c(b)}{4 \sqrt{d(b)}} \sqrt{\log _{b} M}
$$

$$
1-\Phi\left(-\frac{c(b)}{4 \sqrt{d(b)}} \sqrt{\log _{b} M}\right)-O\left(\frac{\sqrt[4]{\log \log _{b} M}}{\sqrt[4]{\log _{b} M}}\right)=1-O\left(\frac{\sqrt[4]{\log \log _{b} M}}{\sqrt[4]{\log _{b} M}}\right)
$$

The event $M^{3 / 4} \leq N<M$ also has probability

$$
1-O\left(\frac{1}{\sqrt[4]{M}}\right)=1-O\left(\frac{\sqrt[4]{\log \log _{b} M}}{\sqrt[4]{\log _{b} M}}\right) .
$$

Therefore it is enough to consider the intersection of these two events, on which

$$
\begin{aligned}
S(N) & >c(b)\left(\log _{b} N-\frac{1}{4} \sqrt{\log _{b} N \log _{b} M}\right) \\
& \geq c(b)\left(\frac{3}{4} \log _{b} M-\frac{1}{4} \sqrt{\log _{b} M \log _{b} M}\right)=\frac{1}{2} c(b) \log _{b} M
\end{aligned}
$$

holds. For every such $N$ we get from (26) that

$$
\begin{aligned}
\int_{0}^{1} \Delta_{N}(x)^{p} \mathrm{~d} x & =S(N)^{p}\left(1+O\left(\frac{1}{\log _{b} M}\right)\right) \\
\left\|\Delta_{N}\right\|_{p} & =S(N)\left(1+O\left(\frac{1}{\log _{b} M}\right)\right)=S(N)+O(b) .
\end{aligned}
$$


Theorem 3 is thus reduced to Theorem 1

Proof of Theorem 4. Similarly to the proof of Theorem 3 we may assume that $p$ is a positive even integer. Since $\left\|\Delta_{N}\right\|_{p}=O\left(b\left(\log _{b} N+1\right)\right)$, by choosing $A$ large enough we may assume that $\lambda \leq \sqrt{\log _{b} M}$. Recall (26) from the proof of Theorem 3 .

$$
\int_{0}^{1} \Delta_{N}(x)^{p} \mathrm{~d} x=S(N)^{p}+O\left(b^{p}\left(\log _{b} N+1\right)^{p-1}\right)
$$

for any $N>0$. Let $N$ be a random variable which is uniformly distributed in $\{0,1, \ldots, M-1\}$. We know from Theorem 2 that $S(N)>\frac{1}{2} c(b) \log _{b} M$ with probability

$$
1-O\left(e^{-c \sqrt[4]{\log _{b} M}}+\frac{1}{b \sqrt{\log _{b} M}-2}\right)
$$

for some constant $c>0$. We also have $\frac{M}{b \sqrt{\log _{b} M}} \leq N<M$ with probability at least $1-O\left(\frac{1}{b \sqrt{\log _{b} M}}\right)$. For all such $N$ we have $\left\|\Delta_{N}\right\|_{p}=S(N)+O(b)$ and

$$
\begin{aligned}
\log _{b} N & =\log _{b} M+O\left(\sqrt{\log _{b} M}\right), \\
\sqrt{\log _{b} N} & =\sqrt{\log _{b} M}+O(1) .
\end{aligned}
$$

These estimates together with Theorem 2 yield

$$
\begin{array}{r}
\frac{1}{M}\left|\left\{0 \leq N<M:\left|\left\|\Delta_{N}\right\|_{p}-\frac{b^{2}-1}{12 b} \log _{b} N\right| \geq A \lambda b \sqrt{\log _{b} N}\right\}\right| \\
=O\left(\frac{\sqrt{\lambda}}{e^{\sqrt{\lambda}-1}-2}+e^{-c \sqrt[4]{\log _{b} M}}+\frac{1}{b^{\sqrt{\log _{b} M}-2}}\right)
\end{array}
$$

for any $\lambda \geq 3$ with some constant $A>0$ depending only on $p$. By replacing $A$ by a larger constant we can simplify the upper bound to $e^{-\sqrt{\lambda}}$ and relax the condition $\lambda \geq 3$ to $\lambda \geq 1$.

Proof of Proposition 5. Let us write $f(x)$ in the form

$$
f(x)=\int_{0}^{1} f(t) \mathrm{d} t+(f(1)-f(0))\left(x-\frac{1}{2}\right)+g(x),
$$


where $g:[0,1] \rightarrow \mathbb{R}$ is defined via (27). Then we have $\int_{0}^{1} g(x) \mathrm{d} x=0$ and $g(0)=g(1)$. Note that (27) is the expansion of $f(x)$ with respect to the Bernoulli polynomials with an explicit remainder term. For any integer $N>0$ we have

$$
\sum_{n=0}^{N-1} f\left(x_{n}\right)=N \int_{0}^{1} f(t) \mathrm{d} t-(f(1)-f(0)) S(N)+\sum_{n=0}^{N-1} g\left(x_{n}\right) .
$$

We now have to show that the last sum is negligible. Since $g$ is twice differentiable on $[0,1]$ and $g(0)=g(1)$, we have that the periodic extension of $g$ to $\mathbb{R}$ with period 1 is Lipschitz, therefore its Fourier series converges to $g$ :

$$
g(x)=\sum_{\ell \in \mathbb{Z}} \hat{g}(\ell) e^{2 \pi i \ell x}
$$

for any $x \in[0,1]$, where

$$
\hat{g}(\ell)=\int_{0}^{1} g(x) e^{-2 \pi i \ell x} \mathrm{~d} x .
$$

We have $\hat{g}(0)=0$, because $\int_{0}^{1} g(x) \mathrm{d} x=0$. Since $g(0)=g(1)$, integration by parts yields that for any integer $\ell \neq 0$

$$
\begin{gathered}
\hat{g}(\ell)=\frac{g^{\prime}(1)-g^{\prime}(0)}{4 \pi^{2} \ell^{2}}-\int_{0}^{1} g^{\prime \prime}(x) \frac{e^{-2 \pi i \ell x}}{4 \pi^{2} \ell^{2}} \mathrm{~d} x=\frac{1}{4 \pi^{2} \ell^{2}} \int_{0}^{1} g^{\prime \prime}(x)\left(1-e^{-2 \pi i \ell x}\right) \mathrm{d} x, \\
|\hat{g}(\ell)| \leq \frac{1}{2 \pi^{2} \ell^{2}} \int_{0}^{1}\left|g^{\prime \prime}(x)\right| \mathrm{d} x=\frac{\left\|f^{\prime \prime}\right\|_{1}}{2 \pi^{2} \ell^{2}} .
\end{gathered}
$$

Therefore

$$
\left|\sum_{n=0}^{N-1} g\left(x_{n}\right)\right|=\left|\sum_{n=0}^{N-1} \sum_{\ell \neq 0} \hat{g}(\ell) e^{2 \pi i \ell x_{n}}\right| \leq \sum_{\ell \neq 0} \frac{\left\|f^{\prime \prime}\right\|_{1}}{2 \pi^{2} \ell^{2}}\left|\sum_{n=0}^{N-1} e^{2 \pi i \ell x_{n}}\right| .
$$

We can split up the sum according to the highest power of $b$ dividing $\ell$. Proposition 10 hence gives

$$
\begin{aligned}
\left|\sum_{n=0}^{N-1} g\left(x_{n}\right)\right| & \leq \sum_{s=0}^{\infty} \sum_{\substack{\ell \neq 0 \\
b^{s} \| \ell}} \frac{\left\|f^{\prime \prime}\right\|_{1}}{2 \pi^{2} \ell^{2}}\left|\sum_{n=0}^{N-1} e^{2 \pi i \ell x_{n}}\right| \\
& \leq \sum_{s=0}^{\infty} \sum_{t \neq 0} \frac{\left\|f^{\prime \prime}\right\|_{1}}{2 \pi^{2} b^{2 s} t^{2}} b^{s+1}=\frac{b^{2}}{6(b-1)}\left\|f^{\prime \prime}\right\|_{1} \leq \frac{b}{3}\left\|f^{\prime \prime}\right\|_{1} .
\end{aligned}
$$




\section{References}

[1] J. Beck: Probabilistic Diophantine Approximation. Randomness in Lattice Point Counting. Springer Monographs in Mathematics. Springer, Cham (2014) xvi+487 pp. ISBN: 978-3-319-10740-0.

[2] Y. S. Chow, H. Teicher: Probability Theory. Independence, Interchangeability, Martingales. Third edition. Springer Texts in Statistics. Springer-Verlag, New York (1997) xxii+488 pp. ISBN: 0-387-98228-0.

[3] M. Drmota, G. Larcher, F. Pillichshammer: Precise distribution properties of the van der Corput sequence and related sequences. Manuscripta Math. 118, no. 1 (2005), 11-41

[4] H. Faure: Discrépances de suites associées à un système de numération (en dimension un). Bull. Soc. Math. France 109, no. 2 (1981), 143-182

[5] L. Kuipers, H. Niederreiter: Uniform Distribution of Sequences. Pure and Applied Mathematics. Wiley-Interscience [John Wiley \& Sons], New York-LondonSydney (1974) xiv+390 pp. ISBN: 0-471-51045-9.

[6] P. Proinov, V. Grozdanov: On the diaphony of the van der Corput-Halton sequence. J. Number Theory 30, no. 1 (1988), 94-104

[7] A. Wohlfarter: Distribution Properties of Generalized van der Corput Sequences (Doctoral dissertation). TU Wien (2009) 